Conclusion Our experience has shown that for the future sustainability of our hospice, this way of working must continue, as we become increasingly reliant on each other. Our recommendation is that collaborative leadership will deliver the ambitions to reach and support more people.

\section{P-274 A HOSPICE LEADERSHIP PIPELINE}

John Knight. LOROS Hospice, Leicester, UK

\subsection{6/bmispcare-2017-hospice.299}

The concept of a 'leadership pipeline' isn't something new (Charan, Drotter, \& Noel, 2001), however, its application to the evolving world of 'hospice' may well be. The pipeline model investigates how we best prepare people for an organisational leadership journey ensuring that they are equipped with and practice leadership skills, well in advance, of any promotion in the organisation. In 2014, following the Hospice UK 'Ambitions' publication, LOROS Hospice set about developing its own leadership pipeline confident of its impact internally and hopeful that it could be of value across the sector. Research shows that several factors influence the development and ongoing practice of leadership. Early exposure to leadership opportunities, learning through 'doing', reflecting upon role models and finally being embedded in a supportive culture are perhaps the main factors noted. Using this knowledge LOROS has developed two leadership interventions. Both are focussed upon 'self' - with the sole aim of helping aspirant and existing leaders invest time to better understand what they personally bring to leadership and just how they impact upon others and how others impact upon them. LOROS believes that this approach, 'to first understand oneself', is a fundamental in effective leadership development and should come before any other form of leadership programme that develops more technical skills such as financial or strategic leadership. 'Aspiring Leaders' is aimed at those new to leadership. It forms an intensive period of time where delegates are challenged to think about how and why they have come to a leadership position and how they wish to be viewed as a leader - nurturing their authentic leadership style. 'Thoughtful Leadership' is aimed at middle management leaders and delves deeper in to their own performance adding skills in managing challenging situations and the impact of coaching as a leadership tool.

\section{P-275 INSPIRING LEADERSHIP LEADING SELF: LEADING WITH OTHERS IN A PALLIATIVE CARE SETTING}

${ }^{1}$ Fiona Wylie, ${ }^{1,2}$ Claire O'Neill, ${ }^{1,3}$ Jane Miller, ${ }^{3}$ Susan Jackson. ${ }^{1}$ Prince and Princess of Wales Hospice, Glasgow, UK; ${ }^{2}$ NHS Greater Glasgow and Clyde Healthboard, Glasgow, UK; ${ }^{3}$ Action for Continuing Care Over Renfrew District (ACCORD) Hospice, Paisley, UK

\subsection{6/bmjspcare-2017-hospice.300}

Background It is recognised within palliative care (PC) that Band 6 Clinical Nurse Specialists (CNS) are working in a time of organisational change with increasing complexity around their specialist role (NHS Education for Scotland 2006). CNSs, in particular newly appointed staff, have reported feeling more vulnerable and stressed and struggling to cope to deliver the same quality of care to patients and their families. To support the above scenario an NHSGGC PC Leadership Steering Group was established in partnership with NES Leadership Unit.

Aims

- Provide a safe space for participants to share and learn from each other

- Provide a training programme which embeds the values, behaviours and attitudes of leadership skills

- Provide a foundation to support greater successional planning throughout palliative care (Scottish Government, 2013; Scottish Government, 2015)

- Increase participants' knowledge and understanding of quality improvement (QI) methodology supporting individual QI action plans.

Methods The development of a 12 month leadership programme based on needs of the participants, open to all Band 6 PC CNSs working within NHSGGC acute and hospice care settings, was established. The programme includes a leadership resource folder, ongoing staff support, feedback to participants utilising 360 degree review and Myers Briggs, provision of mentoring and action learning sets and evaluation process.

Results Interim feedback to date 10 CNSs signed up. To date participants have attended a launch day and two masterclasses held on: Understanding Myers Briggs and working with differences; Understanding Quality Improvement methodology.

All participants have received '360 review process' feedback based on the NHS Scotland Leadership Qualities Framework helping to inform their PDP. All have participated in an action learning set. Three further masterclasses are built around learning needs of the cohort.

Conclusion Feedback to date has highlighted the importance of this programme in addressing a need, focusing on developing leadership skills for a capable, sustainable workforce. Further evaluation is embedded within this programme.

\section{P-276 LEADING CHANGE, ADDING VALUE: POSITIONING NURSING, MIDWIFERY AND CARE STAFF AS LEADERS IN DRIVING CHANGE AND IMPROVING OUTCOMES}

Susan Aitkenhead, Stacey McCann. NHS England, Leeds, UK

\subsection{6/bmispcare-2017-hospice.301}

The presentation will outline the unique leadership role that nursing, midwifery and care staff have in driving change and improving outcomes. It will outline 'Leading Change, Adding Value: a framework for nursing, midwifery and care staff' that was launched in May 2016 by Professor Jane Cummings, Chief Nursing Officer for England. The framework positions nursing, midwifery and care staff as leaders in designing the future of health and care and managing the challenges of today. It explains how staff can demonstrate leadership in reducing the gaps of health and wellbeing, care and quality, and funding and efficiency, whatever their role wherever they work. The framework highlights the need to focus on quality and measurement, which aligns to the national priorities in England's Five Year Forward View - showing why change is needed and what it will look like. The focus is on reducing unwarranted variation, and delivering the 'Triple Aim' of improved outcomes, experience and better use of resources; the benchmarks for quality of services. Unwarranted variation is defined as differences that cannot be justified by geography, demography or infrastructure. The framework offers ten commitments that all staff can make. They align efforts to areas 